\title{
Mazur-Ulam Theorem
}

\author{
Artur Korniłowicz \\ Institute of Informatics \\ University of Białystok \\ Sosnowa 64, 15-887 Białystok, Poland
}

\begin{abstract}
Summary. The Mazur-Ulam theorem [15] has been formulated as two registrations: cluster bijective isometric $\rightarrow$ midpoints-preserving Function of E,F; and cluster isometric midpoints-preserving $\rightarrow$ Affine Function of E,F; A proof given by Jussi Väisälä [23] has been formalized.
\end{abstract}

MML identifier: $\underline{\text { MAZURULM, }}$, version: $\underline{7.11 .07 \quad 4.160 .1126}$

The notation and terminology used in this paper have been introduced in the following papers: [19], [18], [4], [5], [20], [11], [10], [14], [17], [1], [6], [16], [24], [25], [21], [13], [12], [22], [2], [9], [8], [3], and [7].

For simplicity, we use the following convention: $E, F, G$ are real normed spaces, $f$ is a function from $E$ into $F, g$ is a function from $F$ into $G, a, b$ are points of $E$, and $t$ is a real number.

Let us note that $\mathbb{I}$ is closed.

Next we state four propositions:

(1) DYADIC is a dense subset of $\mathbb{I}$.

(2) $\overline{\text { DYADIC }}=[0,1]$.

(3) $a+a=2 \cdot a$.

(4) $(a+b)-b=a$.

Let $A$ be an upper bounded real-membered set and let $r$ be a non negative real number. Observe that $r \circ A$ is upper bounded.

Let $A$ be an upper bounded real-membered set and let $r$ be a non positive real number. Note that $r \circ A$ is lower bounded.

Let $A$ be a lower bounded real-membered set and let $r$ be a non negative real number. Observe that $r \circ A$ is lower bounded. 
Let $A$ be a lower bounded non empty real-membered set and let $r$ be a non positive real number. One can check that $r \circ A$ is upper bounded.

Next we state three propositions:

(5) For every sequence $f$ of real numbers holds $f+(\mathbb{N} \longmapsto t)=t+f$.

(6) For every real number $r$ holds $\lim (\mathbb{N} \longmapsto r)=r$.

(7) For every convergent sequence $f$ of real numbers holds $\lim (t+f)=$ $t+\lim f$.

Let $f$ be a convergent sequence of real numbers and let us consider $t$. One can check that $t+f$ is convergent.

Next we state three propositions:

(8) For every sequence $f$ of real numbers holds $f \cdot(\mathbb{N} \longmapsto a)=f \cdot a$.

(9) $\lim (\mathbb{N} \longmapsto a)=a$.

(10) For every convergent sequence $f$ of real numbers holds $\lim (f \cdot a)=$ $\lim f \cdot a$.

Let $f$ be a convergent sequence of real numbers and let us consider $E, a$. Note that $f \cdot a$ is convergent.

Let $E, F$ be non empty normed structures and let $f$ be a function from $E$ into $F$. We say that $f$ is isometric if and only if:

(Def. 1) For all points $a, b$ of $E$ holds $\|f(a)-f(b)\|=\|a-b\|$.

Let $E, F$ be non empty RLS structures and let $f$ be a function from $E$ into $F$. We say that $f$ is affine if and only if:

(Def. 2) For all points $a, b$ of $E$ and for every real number $t$ such that $0 \leq t \leq 1$ holds $f((1-t) \cdot a+t \cdot b)=(1-t) \cdot f(a)+t \cdot f(b)$.

We say that $f$ preserves midpoints if and only if:

(Def. 3) For all points $a, b$ of $E$ holds $f\left(\frac{1}{2} \cdot(a+b)\right)=\frac{1}{2} \cdot(f(a)+f(b))$.

Let $E$ be a non empty normed structure. Observe that $\operatorname{id}_{E}$ is isometric.

Let $E$ be a non empty RLS structure. Note that $\mathrm{id}_{E}$ is affine and preserves midpoints.

Let $E$ be a non empty normed structure. Observe that there exists a unary operation on $E$ which is bijective, isometric, and affine and preserves midpoints.

Next we state the proposition

(11) If $f$ is isometric and $g$ is isometric, then $g \cdot f$ is isometric.

Let us consider $E$ and let $f, g$ be isometric unary operations on $E$. One can verify that $g \cdot f$ is isometric.

The following proposition is true

(12) If $f$ is bijective and isometric, then $f^{-1}$ is isometric.

Let us consider $E$ and let $f$ be a bijective isometric unary operation on $E$. One can check that $f^{-1}$ is isometric.

We now state the proposition 
(13) If $f$ preserves midpoints and $g$ preserves midpoints, then $g \cdot f$ preserves midpoints.

Let us consider $E$ and let $f, g$ be unary operations on $E$ preserving midpoints. Note that $g \cdot f$ preserves midpoints.

The following proposition is true

(14) If $f$ is bijective and preserves midpoints, then $f^{-1}$ preserves midpoints.

Let us consider $E$ and let $f$ be a bijective unary operation on $E$ preserving midpoints. Observe that $f^{-1}$ preserves midpoints.

Next we state the proposition

(15) If $f$ is affine and $g$ is affine, then $g \cdot f$ is affine.

Let us consider $E$ and let $f, g$ be affine unary operations on $E$. Observe that $g \cdot f$ is affine.

One can prove the following proposition

(16) If $f$ is bijective and affine, then $f^{-1}$ is affine.

Let us consider $E$ and let $f$ be a bijective affine unary operation on $E$. Observe that $f^{-1}$ is affine.

Let $E$ be a non empty RLS structure and let $a$ be a point of $E$. The functor $a$-reflection yields a unary operation on $E$ and is defined as follows:

(Def. 4) For every point $b$ of $E$ holds $a$-reflection $(b)=2 \cdot a-b$.

The following proposition is true

(17) $a$-reflection $\cdot a$-reflection $=\operatorname{id}_{E}$.

Let us consider $E$, $a$. Note that $a$-reflection is bijective.

We now state several propositions:

(18) $a$-reflection $(a)=a$ and for every $b$ such that $a$-reflection $(b)=b$ holds $a=b$.

(19) $a$-reflection $(b)-a=a-b$.

(20) $\| a$-reflection $(b)-a\|=\| b-a \|$.

(21) $a$-reflection $(b)-b=2 \cdot(a-b)$.

(22) $\| a$-reflection $(b)-b\|=2 \cdot\| b-a \|$.

(23) $a$-reflection ${ }^{-1}=a$-reflection.

Let us consider $E, a$. Observe that $a$-reflection is isometric.

Next we state the proposition

(24) If $f$ is isometric, then $f$ is continuous on $\operatorname{dom} f$.

Let us consider $E, F$. Observe that every function from $E$ into $F$ which is bijective and isometric also preserves midpoints.

Let us consider $E, F$. One can check that every function from $E$ into $F$ which is isometric and preserves midpoints is also affine. 


\section{REFERENCES}

[1] Grzegorz Bancerek. The ordinal numbers. Formalized Mathematics, 1(1):91-96, 1990.

[2] Józef Białas. Infimum and supremum of the set of real numbers. Measure theory. Formalized Mathematics, 2(1):163-171, 1991.

[3] Józef Białas and Yatsuka Nakamura. Dyadic numbers and $\mathrm{T}_{4}$ topological spaces. Formalized Mathematics, 5(3):361-366, 1996.

[4] Czesław Byliński. Functions and their basic properties. Formalized Mathematics, 1(1):5565, 1990.

[5] Czesław Byliński. Functions from a set to a set. Formalized Mathematics, 1(1):153-164, 1990.

[6] Czesław Byliński. Partial functions. Formalized Mathematics, 1(2):357-367, 1990.

[7] Czesław Byliński. Some basic properties of sets. Formalized Mathematics, 1(1):47-53, 1990.

[8] Agata Darmochwał. Families of subsets, subspaces and mappings in topological spaces. Formalized Mathematics, 1(2):257-261, 1990.

[9] Agata Darmochwał and Yatsuka Nakamura. Metric spaces as topological spaces - fundamental concepts. Formalized Mathematics, 2(4):605-608, 1991.

[10] Hiroshi Imura, Morishige Kimura, and Yasunari Shidama. The differentiable functions on normed linear spaces. Formalized Mathematics, 12(3):321-327, 2004.

[11] Artur Korniłowicz. Collective operations on number-membered sets. Formalized Mathematics, 17(2):99-115, 2009, doi: 10.2478/v10037-009-0011-0.

[12] Jarosław Kotowicz. Convergent sequences and the limit of sequences. Formalized Mathematics, 1(2):273-275, 1990.

[13] Jarosław Kotowicz. Real sequences and basic operations on them. Formalized Mathematics, 1(2):269-272, 1990.

[14] Rafał Kwiatek. Factorial and Newton coefficients. Formalized Mathematics, 1(5):887-890, 1990.

[15] Stanisław Mazur and Stanisław Ulam. Sur les transformationes isométriques d'espaces vectoriels normés. C. R. Acad. Sci. Paris, (194):946-948, 1932.

[16] Beata Padlewska and Agata Darmochwał. Topological spaces and continuous functions. Formalized Mathematics, 1(1):223-230, 1990.

[17] Jan Popiołek. Real normed space. Formalized Mathematics, 2(1):111-115, 1991.

[18] Andrzej Trybulec. Binary operations applied to functions. Formalized Mathematics, 1(2):329-334, 1990.

[19] Andrzej Trybulec. A Borsuk theorem on homotopy types. Formalized Mathematics, 2(4):535-545, 1991.

[20] Andrzej Trybulec. On the sets inhabited by numbers. Formalized Mathematics, 11(4):341$347,2003$.

[21] Wojciech A. Trybulec. Vectors in real linear space. Formalized Mathematics, 1(2):291-296, 1990.

[22] Zinaida Trybulec. Properties of subsets. Formalized Mathematics, 1(1):67-71, 1990.

[23] Jussi Väisälä. A proof of the Mazur-Ulam theorem. http://www.helsinki.fi/ jvaisala/mazurulam.pdf.

[24] Edmund Woronowicz. Relations and their basic properties. Formalized Mathematics, 1(1):73-83, 1990.

[25] Edmund Woronowicz. Relations defined on sets. Formalized Mathematics, 1(1):181-186, 1990. 